\title{
Interdisciplinaridade na formação de professores: um olhar a partir dos movimentos da produção científica
}

\section{Interdisciplinarity in teacher's training: a look from the movements of scientific production}

\section{Interdisciplinariedad en la formación de profesores: una mirada a partir de los movimientos de la producción científica}

\author{
Aline Souza da $\mathrm{Luz}^{1}$ \\ Maria das Graças C. da S. M. Gonçalves Pinto ${ }^{1}$ \\ DOI: http://dx.doi.org/10.20435/serie-estudos.v23i47.1071
}

\begin{abstract}
Resumo: O trabalho apresenta o resultado de estudo bibliográfico sobre a interdisciplinaridade na formação de professores, realizado em publicações que apresentam um estado da arte sobre a formação de professores no país. No presente trabalho, interessa saber: O que revelam as pesquisas e estudos sobre a interdisciplinaridade na formação de professores? Que aspectos têm sido observados sobre essa temática nesses estudos e pesquisas? Para realização deste estudo bibliográfico, foram consideradas as pesquisas de: André et al. (1999), André (2009), Brzezinski e Garrido (2001), Brzezinski (2009) e Ludke e Goulart (1996). O interesse em realizar o presente trabalho está em sistematizar e analisar o referido tema nas produções, bem como identificar as lacunas de produção de conhecimento sobre a temática. A partir do conjunto de pesquisas analisadas neste estudo bibliográfico, é possível inferir que a interdisciplinaridade, como uma possibilidade de tema de investigação na formação de professores em nível superior, aparece ao longo dos anos nas pesquisas e estudos de forma moderada. Entretanto é preciso verificar que, nos últimos anos, a investigação do tema da interdisciplinaridade na formação de professores tem ganhado uma nova perspectiva devido à implementação das Licenciaturas Interdisciplinares.
\end{abstract}

Palavras-chave: formação de professores; interdisciplinaridade; metanálise.

Abstract: The work presents the result of a bibliographic study about the interdisciplinarity on teacher's training, realized in publications that present a state of art about teacher's training in the country. In the present work it matters to know : What the researches and studies reveal about interdisciplinarity in teacher's training? What aspects have been observed on this theme in those studies and researches? To accomplish this bibliographic study were considered the researches

${ }^{1}$ Universidade Federal de Pelotas (UFPel), Pelotas, Rio Grande do Sul, Brasil. 
from: André et al. (1999), André (2009), Brzezinski e Garrido (2001), Brzezinski (2009) and Ludke e Goulart (1996). The interest in doing the present work is in systematizing and analyzing the mentioned theme in the productions, as well as identifying the knowledge production gaps about the theme. From the set of the analyzed researches in this bibliographic study is possible to infer that the interdisciplinarity, as a possibility of investigation theme in teacher's training in tertiary teaching, appears through the years in researches and studies in a moderate way. However, is necessary to verify that in the past years the theme investigation of interdisciplinary in teacher's training has gained a new perspective due to the implementation of new interdisciplinary teacher's courses.

Keywords: teacher training; interdisciplinarity; metanalysis.

Resumen: El trabajo presenta el resultado de estudio bibliográfico sobre la interdisciplinariedad en la formación de profesores, realizado en publicaciones que presentan un estado del arte sobre la formación de profesores en Brasil. En el presente trabajo interesa saber: ¿Qué revelan las investigaciones y estudios sobre la interdisciplinariedad en la formación de profesores? ¿Qué aspectos han sido observados sobre esta temática en esos estudios e investigaciones? Para la realización de este estudio bibliográfico se consideraron las investigaciones de: André et al. (1999), André (2009), Brzezinski y Garrido (2001), Brzezinski (2009) y Ludke y Goulart (1996). El interés en realizar el presente trabajo está en sistematizar y analizar el referido tema en las producciones, así como identificar las lagunas de producción de conocimiento sobre la temática. A partir del conjunto de investigaciones analizadas en ese estudio bibliográfico es posible inferir que la interdisciplinaridad, como una posibilidad de tema de investigación en la formación de profesores a nivel superior, aparece a lo largo de los años en las investigaciones y estudios de forma moderada. Sin embargo, es necesario verificar que en los últimos años la investigación del tema de la interdisciplinariedad en la formación de profesores em Brasil ha ganado una nueva perspectiva debido a la implementación de las Licenciaturas Interdisciplinares.

Palabras clave: formación de profesores; interdisciplinariedad; metanálisis.

\section{INTRODUÇÃO}

Formação de professores é uma área do conhecimento que, ao longo dos anos, tem apresentado diversas possibilidades de investigação. Alguns temas têm sido mais frequentes nas pesquisas e estudos, como o caso das temáticas sobre configuração identitária profissional, os saberes necessários para essa formação, o trabalho dos professores, entre outros.

A interdisciplinaridade como uma possibilidade de tema de investigação da formação de professores em nível superior aparece de forma moderada, ao longo dos anos, nas pesquisas e estudos.

Cabe esclarecer que não é intenção discutir o conceito de interdisciplinaridade, visto que, no presente trabalho, interessa saber: O que revelam as pesquisas e estudos sobre a interdisciplinaridade na formação de professores? Que aspectos têm sido observados sobre esta temática nesses estudos e pesquisas? O interesse 
em realizar o presente trabalho está em sistematizar e analisar o referido tema nas produções, bem como identificar as lacunas de produção de conhecimento sobre a temática.

Com o propósito de responder a essas perguntas, inicialmente expõe-se a metodologia utilizada. Em seguida, são apresentados os dados coletados e analisados e, por fim, as considerações finais.

\section{CAMINHO METODOLÓGICO}

O presente trabalho constitui-se num estudo bibliográfico, organizado no tipo metanálise. Tanto Brzezinski (2009) como Gatti (2014) nomeiam de metanálise o processo de revisar estudos e pesquisas que já fizeram um mapeamento sobre o tema. Trata-se em suma, de efetivar um estudo a partir dos diferentes trabalhos que realizam um Estado da Arte sobre a formação de professores no Brasil.

É importante ressaltar que esse tipo de estudo é importante, pois sistematiza as diferentes análises já realizadas e dispersas e fragmentadas nos diferentes espaços de produção científica.

Ademais, Gatti (2014, p. 28) também ressalta a importância desse tipo de estudo: "É preciso considerar, no entanto, que são escassas, senão inexistentes, sínteses de conhecimento acumulado sobre temas básicos em educação que sejam acessíveis em sua linguagem e porte".

Segundo as autoras Romanowski e Ens (2006), o aumento da produção científica $^{2}$ na área da educação, na forma de trabalhos e pesquisas de teses e dissertações, de trabalhos apresentados em eventos da área, das publicações em periódicos, requer constantemente um balanço dessa produção. Assim, afirmam que faltam estudos dessa ordem.

[...] pode-se dizer que faltam estudos que realizem um balanço e encaminhem para a necessidade de um mapeamento que desvende e examine o conhecimento já elaborado e apontem os enfoques, os temas mais pesquisados e as lacunas existentes. (ROMANOSKI; ENS, 2006, p. 38).

Esclarecido o que são estudos tipo "Metanálise", caberia ainda a pergunta: Qual a diferença entre as pesquisas tipo "Estado da Arte" e as pesquisas do

2 Impulsionado pela política de avaliação da Coordenação de Aperfeiçoamento de Pessoal de Nível Superior (CAPES). 
tipo "Metanálise"? As pesquisas tipo "Estado da Arte" realizam investigações contemplando diferentes espaços da produção científica, tais como: eventos científicos, periódicos, teses, dissertações, entre outros, assim como os estudos tipo "Metanálise". Entretanto o que difere um tipo de pesquisa de outra é quanto ao "corpus de análise". As pesquisas tipo "Estado da Arte" utilizam como fonte de seu "corpus de análise" os diferentes espaços da produção científica, gerando uma síntese do conhecimento produzido sobre um determinado tema. Já as pesquisas tipo "Metanálise" utilizam como "corpus de análise" os estudos tipo "Estado da Arte", ou seja, utilizam diferentes sínteses já realizadas, entretanto partem de um outro olhar analítico, buscado nas diferentes sínteses, pontos em comum, divergências, lacunas, dentre outros aspectos.

Dessa forma, o presente trabalho procura sistematizar e analisar o referido tema nos diferentes espaços da produção científica especializada, identificando os movimentos da produção do conhecimento científico sobre a interdisciplinaridade na formação de professores. Além disso, busca identificar as lacunas de produção de conhecimento sobre a temática. Dito de outra forma, o presente trabalho revisita os estudos que realizaram um Estado da Arte sobre a formação de professores no Brasil, procurando indicativos de como a interdisciplinaridade tem aparecido e como tem sido discutida nesses estudos.

Parte-se do exame do conjunto de estudos e de pesquisas sobre formação de professores, a partir da realidade brasileira, os quais mapearam teses e dissertações dos programas de pós-graduação em educação; trabalhos apresentados na reunião da Associação Nacional de Pós-Graduação e Pesquisa em Educação (ANPED) e publicações em periódicos da área. Foram considerados os estudos e pesquisas de André et al. (1999), André (2009), Brzezinski e Garrido (2001), Brzezinski (2009) e Ludke e Goulart (1996).

Consideramos esses estudos, por terem estes sido referência para outros trabalhos e por sua relevância no Estado da Arte sobre a formação de professores no país.

A metodologia de análise dos trabalhos incide numa "reconciliação integrativa", que, segundo André et al. (1999, p. 308 ), "Consiste num balanço do conhecimento, baseado na análise comparativa de vários trabalhos, sobre uma determinada temática". 


\section{OS MOVIMENTOS DA PRODUÇÃO CIENTÍFICA E ALGUMAS ANÁLISES}

É possível encontrar estudos e pesquisas sobre a interdisciplinaridade desde a década de $1970^{3}$. Esses estudos indicam que as primeiras pesquisas buscavam discutir seu conceito, bem como verificar como se dava sua materialização na prática do professor realizada na escola básica. Tal indício revela que foram estudos que investigaram a interdisciplinaridade no contexto posterior à formação do professor. Esse tipo de análise permanece até os dias atuais com um volume consistente de investigações.

A partir dos anos 1980, com alguns poucos trabalhos, a interdisciplinaridade começava a ser investigada no processo de formação de professores e teve, a partir da década de 1990, um adensamento nesses estudos. A fim de aprofundar essa análise, tomaremos os trabalhos anunciados acima: André et al. (1999), André (2009), Brzezinski e Garrido (2001), Brzezinski (2009) e Ludke e Goulart (1996).

O estudo analítico realizado por André et al. (1999) investigou diferentes espaços de produção científica, da década de 1990, os quais tratavam da formação de professores: teses e dissertações; periódicos e os trabalhos apresentados no GT 8 da ANPED. Dentre outros achados, concluem que, de maneira geral, a produção científica sobre a formação de professores concentra-se em três categorias de análise: formação inicial, formação continuada e identidade e profissionalização docente.

Ao revisitar esse trabalho, procurando evidências sobre a temática, a interdisciplinaridade é citada como um dos aspectos investigados dentre os 27 artigos que tratam da formação inicial, num total de 115 artigos investigados nos periódicos. Não houve incidência da temática interdisciplinaridade nas teses e dissertações, do período de 1990 a 1998. A análise sobre os trabalhos do GT 8 será retomada posteriormente.

Num trabalho posterior, André (2009) se deteve em analisar somente a produção discente dos programas de pós-graduação em educação do período entre 1999-2003. Entre as 255 pesquisas sobre formação inicial, 115 trataram dos cursos de licenciatura. Dentre os aspectos investigados desses cursos, a interdisciplinaridade $^{4}$ aparecia como um dos temas investigados pelos discentes. Na categoria

\footnotetext{
${ }^{3}$ Com as primeiras pesquisas de Ivani Fazenda.

${ }^{4}$ Neste estudo a autora não aponta sob que circunstância aparece a interdisciplinaridade nos cursos.
} 
formação continuada, que abarcou 111 trabalhos, a interdisciplinaridade apareceu como um dos aspectos investigados nas práticas pedagógicas dos professores.

Sobre a interdisciplinaridade, André (2009, p. 49) faz a seguinte observação:

Nos anos mais recentes [...] surgem estudos sobre interdisciplinaridade tanto na licenciatura, quanto no magistério do ensino médio, nos projetos e nas práticas de formação continuada. São estudos que defendem a perspectiva interdisciplinar, mas que raramente relatam situações em que esteja sendo efetivamente implantada.

Ao estabelecer uma comparação entre os dois estudos de André et al. (1999) e André (2009), na tentativa de compreender como a interdisciplinaridade aparece como objeto de pesquisa da produção discente (teses e dissertações), o primeiro estudo revela que, na década de 1990, não houve incidência de estudo da temática, embora, nesse mesmo período, ela seja temática de análises em artigos de periódicos. O segundo estudo indica que começaram a surgir pesquisas, no início dos anos 2000, sobre a interdisciplinaridade como elemento da formação de professores.

Sobre os trabalhos apresentados no GT 8 da ANPED que trata da formação docente, dois estudos sistematizam as análises: Brzezinski e Garrido (2001) e Brzezinski (2009). A primeira revisão analisou 70 trabalhos no período entre 19921998. Dos 70 trabalhos, 29 tratavam da formação inicial e destes somente quatro trabalhos abordavam a temática da interdisciplinaridade ${ }^{5}$. Os temas abordados sobre a interdisciplinaridade nesses quatro trabalhos versavam sobre a proposição de um currículo interdisciplinar e a interdisciplinaridade, como princípio para estabelecer relações entre as disciplinas das áreas específicas e pedagógicas, e como articuladora das disciplinas teóricas e práticas através de disciplinas integradoras e eixos interdisciplinares. Ainda dos 70 trabalhos analisados, 11 tratavam da profissionalização docente. Nessa categoria, somente um trabalho discutiu o tema da interdisciplinaridade como uma prática no processo formativo.

A segunda revisão realizada por Brzezinski (2009) abarcou os trabalhos do GT 8 da ANPED, no período entre 1999-2008. Esse estudo menciona o surgimento, ainda muito incipiente, da temática entre os 118 trabalhos investigados. Especificamente na categoria Identidade e Profissionalização Docente, estavam

\footnotetext{
${ }^{5}$ Esses trabalhos foram citados inicialmente no estudo de Brzezinski e Garrido (2001).
} 
26 trabalhos e uma das temáticas investigadas foi "práticas interdisciplinares na Pedagogia".

A revisão realizada por Ludke e Goulart (1996) abrangeu 20 periódicos da área, levantando mais de 65 artigos tratando das Licenciaturas, entre o final dos anos 1980 e início dos 1990. A discussão sobre a interdisciplinaridade e formação de professores apresentou uma variedade de posições sobre o assunto.

Num esforço de síntese das discussões percebidas, Ludke e Goulart (1996, p. 34) afirmam: "A solução via integração/interdisciplinaridade surge na literatura analisada como tentativa de superar as várias fragmentações e rupturas registradas hoje nos cursos de formação de professores".

As autoras apontam que o problema de fragmentação e ruptura registrados nos cursos de formação de professores anunciavam a desarticulação que havia entre os conteúdos de uma mesma disciplina; entre as disciplinas do currículo; entre as disciplinas de conhecimento específico e de conhecimento pedagógico e também a desarticulação entre os departamentos e institutos (LUDKE; GOULART, 1996).

Nesse contexto, a integração/interdisciplinaridade foi tomada como uma solução para o problema de fragmentação dos cursos. Como podemos perceber, os problemas apontados referem-se mais exatamente a problemas de fragmentação "curricular" dos cursos. De maneira geral, as autoras apontam que a integração/ interdisciplinaridade se restringiu então à reformulação curricular. Ou seja, ao elegerem a integração/interdisciplinaridade como proposta para a reformulação curricular, os cursos buscavam superar a fragmentação curricular, seja entre os conteúdos da disciplina, seja entre as disciplinas do currículo. Verifica-se que os conceitos de integração e interdisciplinaridade eram tomados como sinônimos.

Da revisão feita por Ludke e Goulart (1996), ficam os apontamentos de que a interdisciplinaridade era discutida na literatura da área como uma solução ao problema de fragmentação dos cursos de formação de professores.

Feita a exposição mais detalhada dos estudos que embasaram o presente trabalho, a seguir apresentamos um quadro síntese da análise. 
Quadro 1 - Síntese Analítica da Produção

\begin{tabular}{|c|c|c|c|c|}
\hline $\begin{array}{l}\text { Trabalho } \\
\text { analisado }\end{array}$ & $\begin{array}{c}\text { Período } \\
\text { analisado }\end{array}$ & Objeto & $\begin{array}{l}\text { Categorias } \\
\text { analisadas }\end{array}$ & $\begin{array}{c}\text { Sobre a } \\
\text { interdisciplinaridade }\end{array}$ \\
\hline \multirow[t]{2}{*}{$\begin{array}{l}\text { André et al. } \\
\text { (1999) }\end{array}$} & $\begin{array}{l}\text { Década de } \\
1990\end{array}$ & $\begin{array}{l}115 \text { artigos } \\
\text { investigados } \\
\text { nos periódicos }\end{array}$ & $\begin{array}{l}27 \text { artigos que } \\
\text { tratavam da } \\
\text { formação inicial }\end{array}$ & $\begin{array}{l}\text { A temática pareceu } \\
\text { citada como elemento } \\
\text { importante no processo } \\
\text { de formação, sem quanti- } \\
\text { ficação de incidências ou } \\
\text { outros detalhes. }\end{array}$ \\
\hline & 1990-1998 & $\begin{array}{l}\text { teses e } \\
\text { dissertações }\end{array}$ & & $\begin{array}{l}\text { Não houve incidência da } \\
\text { temática. }\end{array}$ \\
\hline \multirow[t]{2}{*}{$\begin{array}{l}\text { André } \\
\text { (2009) }\end{array}$} & \multirow[t]{2}{*}{ 1999-2003 } & \multirow[t]{2}{*}{$\begin{array}{l}\text { teses e } \\
\text { dissertações }\end{array}$} & $\begin{array}{l}255 \text { pesquisas } \\
\text { estavam na cate- } \\
\text { goria formação } \\
\text { inicial, dessas } 115 \\
\text { pesquisas sobre } \\
\text { licenciaturas }\end{array}$ & \multirow{2}{*}{$\begin{array}{l}\text { A temática apareceu } \\
\text { como um aspecto inves- } \\
\text { tigado, sem quantificação } \\
\text { de incidências ou outros } \\
\text { detalhes. }\end{array}$} \\
\hline & & & \begin{tabular}{|l}
11 pesquisas na \\
categoria forma- \\
ção continuada \\
\end{tabular} & \\
\hline \multirow{3}{*}{$\begin{array}{l}\text { Brzezinski; } \\
\text { Garrido } \\
\text { (2001) }\end{array}$} & \multirow{3}{*}{ 1992-1998 } & \multirow{3}{*}{$\begin{array}{l}70 \text { trabalhos } \\
\text { analisados do } \\
\text { GT } 8 \text { da ANPED }\end{array}$} & & $\begin{array}{l}\text { Um estudo do curso de } \\
\text { pedagogia - a interdis- } \\
\text { ciplinaridade aparece } \\
\text { como organização } \\
\text { curricular }\end{array}$ \\
\hline & & & $\begin{array}{l}29 \text { textos tratavam } \\
\text { da formação inicial }\end{array}$ & $\begin{array}{l}\text { Entre os } 17 \text { estudos que } \\
\text { investigam as Licencia- } \\
\text { turas, a interdisciplina- } \\
\text { ridade apareceu como } \\
\text { organização curricular e } \\
\text { na relação entre discipli- } \\
\text { nas de áreas específicas } \\
\text { e pedagógicas, e como } \\
\text { articuladora das discipli- } \\
\text { nas teóricas e práticas }\end{array}$ \\
\hline & & & $\begin{array}{l}11 \text { trabalhos trata- } \\
\text { vam da profissio- } \\
\text { nalização docente }\end{array}$ & $\begin{array}{l}\text { Somente um trabalho } \\
\text { discutiu a interdisciplina- } \\
\text { ridade como uma prática } \\
\text { no processo formativo. }\end{array}$ \\
\hline
\end{tabular}




\begin{tabular}{|l|l|l|l|l|}
\hline $\begin{array}{c}\text { Trabalho } \\
\text { analisado }\end{array}$ & $\begin{array}{c}\text { Período } \\
\text { analisado }\end{array}$ & \multicolumn{1}{c|}{ Objeto } & $\begin{array}{c}\text { Categorias } \\
\text { analisadas }\end{array}$ & $\begin{array}{c}\text { Sobre a } \\
\text { interdisciplinaridade }\end{array}$ \\
\hline $\begin{array}{l}\text { Brzezinski } \\
(2009)\end{array}$ & $1999-2008$ & $\begin{array}{l}118 \text { trabalhos } \\
\text { investigados } \\
\text { no GT } 8-\end{array}$ & $\begin{array}{l}\text { 26 trabalhos es- } \\
\text { tavam na catego- } \\
\text { ria Identidade e } \\
\text { Profissionalização } \\
\text { Docente }\end{array}$ & $\begin{array}{l}\text { A temática apareceu } \\
\text { como um aspecto inves- } \\
\text { tigado, sem quantificação } \\
\text { de incidências, ou outros } \\
\text { detalhes. }\end{array}$ \\
\hline $\begin{array}{l}\text { Ludke; } \\
\text { Goulart } \\
\text { (1996) }\end{array}$ & $\begin{array}{l}\text { Final dos } \\
\text { anos } 80 \text { e } \\
\text { início dos } \\
90\end{array}$ & $\begin{array}{l}20 \text { periódicos, } \\
\text { analisando } \\
\text { mais de } 65 \\
\text { artigos }\end{array}$ & $\begin{array}{l}\text { A interdisciplinaridade } \\
\text { aparece, na literatura da } \\
\text { aroblemas das } \\
\text { licenciaturas } \\
\text { ao problema de fragmen- } \\
\text { tação curricular dos cur- } \\
\text { sos de formação de pro- } \\
\text { fessores. Nesse sentido, } \\
\text { a interdisciplinaridade } \\
\text { restringiu-se a uma pro- } \\
\text { posta de reformulação } \\
\text { curricular. }\end{array}$ \\
\hline
\end{tabular}

Fonte: elaboração das autoras.

Os diferentes corpus de análise que compõem os estudos, quais sejam, teses e dissertações, artigos de periódicos e trabalhos apresentados no GT 8 da ANPED, indicam de maneira geral que os estudos e as pesquisas sobre a interdisciplinaridade, como objeto de pesquisa no processo de formação inicial em nível superior de professores, aparecem como tema de pesquisa ou de discussão de forma relativamente escassa, se comparado a outros temas. Na produção discente, a interdisciplinaridade apareceu como temática de pesquisa somente por volta do início dos anos 2000.

Os primeiros estudos sobre interdisciplinaridade que datam da década de 1970 buscavam verificar como a interdisciplinaridade era materializada na prática do professor realizada na escola básica. A interdisciplinaridade era analisada num processo posterior à formação do professor. É preciso lembrar que a formação do professor ocorria de forma disciplinar e fundada numa racionalidade técnico-instrumental ${ }^{6}$. Dessa forma, discutir práticas interdisciplinares sem pensar numa

\footnotetext{
${ }^{6}$ Modelo de formação de professores em que a formação pedagógica ocorre na sequência posterior à formação dos conhecimentos específicos. A formação pedagógica é baseada no ensino de métodos e técnicas de ensino.
} 
formação interdisciplinar é compreender a interdisciplinaridade como uma técnica de ensino. A interdisciplinaridade nesse contexto é compreendida como um princípio metodológico.

Já por volta do final dos anos 1980 e início dos 1990, a interdisciplinaridade aparece como tema nos periódicos da área, como proposta de reformulação curricular dos cursos de licenciatura.

Também nas análises, foi possível observar que a interdisciplinaridade passou a ser investigada como um elemento do processo de formação do professor, geralmente relacionada a experiências isoladas entre professores e componentes curriculares dos cursos de formação inicial. Cabe salientar que todas as discussões sobre a interdisciplinaridade nos diferentes espaços da produção científica ocorreram a partir de cursos disciplinares de formação de professores.

O exame desse conjunto de estudos revela que a interdisciplinaridade aparece como como princípio metodológico nas práticas pedagógicas (posterior à formação); como proposta de reformulação curricular, mais como um conceito operacional no processo de formação e como um elemento desse processo, em práticas e experiências isoladas nos cursos disciplinares.

Mais recente, encontraram-se mais estudos que analisam experiências isoladas de interdisciplinaridade na formação inicial de professores, como por exemplo, o estudo de Silva (2014), que analisou a prática interdisciplinar realizada em três componentes curriculares do curso de Licenciatura em Letras. Já Pierson (2008) analisou a prática interdisciplinar realizada entre dois componentes curriculares e entre duas licenciaturas: Biologia e Física. Em Mateiro e Ferreira (2013), as autoras consideraram como uma prática interdisciplinar a componente curricular de Estágio Curricular ofertada para duas licenciaturas: Música e Teatro. O estudo mais denso foi de Teixeira et al. (2014). Os autores buscaram encontrar indícios de práticas interdisciplinares nos currículos de 10 licenciaturas em Matemática do Brasil. Foram contabilizados 387 componentes curriculares e desses, segundo as autoras, somente $2 \%$ possuem caráter interdisciplinar. Esse estudo foi motivado pela necessidade dos autores em verificar o quanto das normativas legais, na forma das Diretrizes Curriculares para Formação de Professores (DCNFP) (BRASIL, 2002) ou os próprios Parâmetros Curriculares Nacionais (PCNs) (BRASIL, 1998), estavam em implementação nos cursos, visto que esses documentos anunciam a proposição da interdisciplinaridade. 
Como é possível observar, são mais estudos que seguem na análise de experiências isoladas de interdisciplinaridade na formação inicial de professores, visto serem experiências de alguns professores e com alguns componentes curriculares desses cursos.

Entretanto é preciso anunciar que, nos últimos anos, a investigação do tema da interdisciplinaridade na formação de professores tem ganhado uma nova perspectiva devido à implementação das Licenciaturas Interdisciplinares. A diferença dessas investigações mais recentes sobre o tema para os outros estudos citados anteriormente é de que os primeiros são investigações sobre "novos" cursos que se denominam interdisciplinares, enquanto os últimos são práticas e experiências realizadas em cursos disciplinares.

No sentido de continuar identificando o movimento das pesquisas sobre a interdisciplinaridade na formação de professores, a pesquisa realizada por Alves e Araújo (2015), examinando o Banco de Teses e Dissertações da Coordenação de Aperfeiçoamento de Pessoal de Nível Superior (CAPES), procurou encontrar discussões em torno da formação inicial de professores interdisciplinares. A partir da palavra interdisciplinaridade, as autoras obtiveram um total de 516 trabalhos. Desses, somente 12 apresentam relação com o assunto de formação inicial de professores interdisciplinares e foram divididos em três categorias de análise: Concepções, Políticas educacionais e Propostas didáticas. Nos achados das autoras, a categoria "concepções" absorveu quatro trabalhos que buscavam investigar a concepção de interdisciplinaridade veiculada nos cursos analisados. Já a categoria "politicas educacionais", absorveu dois trabalhos, sendo um repetido da categoria anterior. Os trabalhos da categoria "politicas educacionais" investigaram a contribuição das políticas educacionais no fomento às práticas interdisciplinares. A categoria que mais agregou trabalhos foi "propostas didáticas", com sete trabalhos, em que analisaram projetos realizados nos cursos e implementações de práticas, entre outros.

A conclusão das autoras revela uma lacuna de produção de conhecimentos sobre a temática,

[...] apesar dos trabalhos estarem relacionados à formação inicial e a interdisciplinaridade, nenhum trabalho pesquisou realmente sobre a formação inicial de professores interdisciplinares, ou seja, de como esses estão se constituindo interdisciplinares a partir da inserção e formação nos cursos. (ALVES; ARAÚJO, 2015, p. 6). 
As implicações da ausência de pesquisas sobre essa questão limita a construção de argumentos e conhecimentos consistentes sobre a Formação de Professores. A constituição de um campo de conhecimentos e pesquisa sobre a formação de professores legitima um conjunto de conhecimentos consistentes sobre o campo e, com isso, contribui para valorização da formação e profissão docente, pois evidencia que é uma profissão com saberes e conhecimentos próprios.

\section{CONSIDERAÇÕES FINAIS}

A efetivação de estudos tipo metanálise (BRZEZINSKI, 2009; GATTI, 2014) ou o balanço da produção da área (ROMANOSKI; ENS, 2006) são fundamentais, pois sintetizam a produção do conhecimento da área que, muitas vezes, se encontra dispersa e fragmentada nos diferentes espaços de produção científica.

Ademais, esses mapeamentos são importantes para acompanhar o processo do desenvolvimento do campo de conhecimento, além de fornecer elementos para aperfeiçoar a pesquisa no campo do saber (ANDRÉ, 2009).

Este trabalho procurou responder às questões: O que revelam as pesquisas e estudos sobre a interdisciplinaridade na formação de professores? Que aspectos têm sido observados sobre essa temática nesses estudos e pesquisas? Para isso apresentou o resultado do estudo bibliográfico sobre a interdisciplinaridade na formação de professores, do tipo "Metanálise". A reconciliação integrativa entre os trabalhos de André et al. (1999), André (2009), Brzezinski e Garrido (2001), Brzezinski (2009) e Ludke e Goulart (1996) oferece uma variedade de posições sobre o assunto, bem como as lacunas de produção de conhecimento sobre o tema.

A sistematização e a análise dos dados revelam que a interdisciplinaridade tem sido analisada nas práticas pedagógicas realizadas pelo professor da escola básica (posterior à formação) e no processo de formação (como um elemento desse processo) em práticas e experiências isoladas nos cursos disciplinares. Entretanto é preciso verificar que, nos últimos anos, a investigação do tema da interdisciplinaridade na formação de professores tem ganhado uma nova perspectiva devido à implementação das Licenciaturas Interdisciplinares. A diferença dessas investigações mais recentes sobre o tema para os outros estudos citados anteriormente é de que os primeiros são investigações sobre "novos" cursos que se denominam interdisciplinares, enquanto os últimos são práticas e experiências realizadas em cursos disciplinares. 
A partir do conjunto de pesquisas analisadas neste estudo bibliográfico, é possível inferir que a interdisciplinaridade, como uma possibilidade de tema de investigação na formação de professores em nível superior, tem aparecido de forma incipiente nas pesquisas e estudos dos últimos anos.

Esse conjunto de estudos e pesquisas analisados até aqui auxilia a compreender os movimentos da produção do conhecimento científico sobre a interdisciplinaridade na formação de professores em nível superior.

O debate não está encerrado. A pouca produção identificada impele a necessidade de mais estudos sobre a temática. Assim, ao finalizar, sugere-se a necessidade de outros estudos, que se debrucem sobre as tantas inquietações aqui apresentadas.

\section{REFERÊNCIAS}

ALVES, C. C.; ARAÚJO, R. R. Estado da arte da formação inicial de professores interdisciplinares no Banco de teses e dissertações da CAPES. In: CONGRESSO INTERNACIONAL DE EDUCAÇÃO CIENTÍFICA E TECNOLÓGICA, 3., 2015, Santo Ângelo, RS. Anais eletrônicos... Santo Ângelo, RS: URI- Santo Ângelo, 2015. Disponível em: <http://santoangelo.uri.br/ ciecitec2015/>. Acesso em: 20 ago. 2015.

ANDRÉ, M. et al. Estado da arte da formação de professores no Brasil. Educação \& Sociedade, Campinas, SP, ano XX, n. 68, p. 301-9, dez. 1999 Disponível em: <www.scielo. br/pdf/es/v20n68/a15v2068.pdf>. Acesso em: 18 maio 2015.

ANDRÉ, M. A produção acadêmica sobre formação de professores: um estudo comparativo das dissertações e teses defendidas nos anos 1990 e 2000. Revista Brasileira de Pesquisa sobre Formação Docente, Belo Horizonte, v. 1, n. 1, p. 41-56, ago./dez. 2009. Disponível em: <http://formacaodocente.autenticaeditora.com.br/artigo/exibir/1/7/1>. Acesso em: 18 maio 2015.

BRASIL. Conselho Nacional de Educação. Ministério da Educação. Resolução CNE/CP n. 1, de 18 de fevereiro de 2002. Diretrizes Curriculares Nacionais para a Formação de Professores da Educação Básica, em nível superior, curso de licenciatura, de graduação plena. Disponível em: <http://portal.mec.gov.br/cne/arquivos/pdf/rcp01_02.pdf>.

. Presidência da República. Ministério da Educação. Secretaria de Educação Fundamental. Parâmetros Curriculares Nacionais: Ciências da Natureza. Terceiro e Quarto Ciclos. Brasília: MEC, 1998.

BRZEZINSKI, I. Pesquisa sobre formação de profissionais da educação no GT 8/Anped: travessia histórica. Revista Brasileira de Pesquisa sobre Formação Docente, Belo Horizonte, v. 1, n. 1, p. 71-94, ago./dez. 2009. Disponível em <http://formacaodocente. autenticaeditora.com.br/artigo/exibir/1/6/1>. Acesso em: 18 maio 2015. 
BRZEZINSKI, I.; GARRIDO, E. Análise dos trabalhos do GT Formação de professores: o que revelam as pesquisas do período 1992-1998. Revista Brasileira de Educação, Rio de Janeiro, n. 18, p. 82-100, set./dez. 2001. Disponível em: <http://www.scielo.br/pdf/ rbedu/n18/n18a08.pdf>. Acesso em: 24 maio 2015.

GATTI, B. A. Formação inicial de professores para a Educação Básica: pesquisas e políticas educacionais. Estudos em Avaliação Educacional, São Paulo, v. 25, n. 57, p. 24-54, jan./abr. 2014. Disponível em: <www.fcc.org.br/pesquisa/publicacoes/eae/arquivos/1899/1899. pdf>. Acesso em: 22 jun. 2015.

LUDKE, M.; GOULART, S. M. Licenciatura - novos caminhos pela via da interdisciplinaridade. In: BRZEZINSKI, I. (Org.). Formação de professores: um desafio. Goiania: UGC, 1996. p. 29-44.

MATEIRO, T.; FERREIRA, M. S. Interdisciplinaridade na formação de professores de música e teatro. In: SIMPÓSIO INTERNACIONAL SOBRE INTERDISCIPLINARIDADE NO ENSINO, NA PESQUISA E NA EXTENSÃO - REGIÃO SUL, SIIEPE - Sul 2013, Florianópolis. Anais eletrônicos... Florianópolis: EGC/UFSC, 2013. Disponível em: <http://www.siiepe.ufsc.br/wp-content/uploads/2013/10/B-Mateiro.pdf>. Acesso em: 31 jul. 2015.

PIERSON, A. H. C. et al. Uma experiência interdisciplinar na formação inicial de professores. Revista Interacções, Braga, Portugal, v. 4, n. 9, p. 113-28, 2008. Disponível em: <http://revistas.rcaap.pt/interaccoes/article/view/363/318>. Acesso em: 15 jul. 2015.

ROMANOWSKI, Joana Paulin; ENS, Romilda Teodora. As pesquisas denominadas do tipo "Estado da Arte" em educação. Revista Diálogo Educacional, Curitiba, v. 6, n. 19, p.37-50, set./dez. 2006. Disponível em: <www2.pucpr.br/reol/index.php/ DIALOGO?dd1=237>. Acesso em: 15 jul. 2015.

SILVA, F. O. Prática interdisciplinar na formação do professor de Língua Portuguesa. In: SEMINÁRIO INTERNACIONAL DA REDESTRADO, 10., 2014, Salvador. Anais eletrônicos... Salvador: REDESTRADO, 2014. CD-ROM.

TEIXEIRA, A. S. et al. A prática do trabalho interdisciplinar nos currículos de cursos de licenciatura em matemática. In: MOSTRA DA PRODUÇÃO UNIVERSITÁRIA, 13., 2014, Rio Grande, RS. Anais eletrônicos... Rio Grande, RS: Editora da FURG, 2014. Disponível em: <www.propesp.furg.br/anaismpu/cd2014/cic/607.doc>. Acesso em: 31 jul. 2015. 


\section{Sobre as autoras:}

Aline Souza da Luz: Doutoranda do Programa de Pós-Graduação em Educação da Universidade Federal de Pelotas, Rio Grande do Sul. Mestre em Educação e Especialista em Coordenação Pedagógica pela Universidade Federal do Rio Grande do Sul (UFRGS). Pedagoga, graduada pela Universidade Luterana do Brasil (ULBRA). E-mail: alineluz.ufrgs@gmail.com

Maria das Graças C. da S. M. Gonçalves Pinto: Doutora em Educação pela Universidade do Vale do Rio dos Sinos. Mestre em Educação pela Universidade Federal de Santa Maria. Pedagoga pela Universidade Federal de Uberlândia. Professora da Faculdade de Educação e do Programa de Pós-Graduação em Educação da Universidade Federal de Pelotas. E-mail: profgra@gmail.com

\section{Recebido em maio de 2017}

Aprovado em outubro de 2017 
